\title{
A Prospective Study Estimating the Mupirocin Resistance in Health Care Providers with MRSA Nasal Carriage in a Tertiary Care Hospital in Rural South India
}

\author{
K.T. Sangeetha, S. Sreeja* and Ruby Thomas \\ Department of Microbiology, Akash Institute of Medical Sciences and Research Center, \\ Prasannahalli road, Devanahalli, Bengaluru rural-562110, Karnataka, India \\ *Corresponding author
}

\section{A B S T R A C T}

\begin{abstract}
This study was conducted to estimate the prevalence of nasal carriage of Methicillin resistant Staphylococcus aureus (MRSA) and its Mupirocin resistance in health care providers (HCPs). Sterile swabs from the anterior nares of the HCPs were collected and then transported to the Microbiology laboratory for processing. The Staphylococcus aureus (S.aureus) isolates were tested for Methicillin resistance on Mueller Hinton agar using Cefoxitin disc (30ug) by Kirby Bauer disc diffusion method. A zone of $\leq 21 \mathrm{~mm}$ was considered as MRSA. All the MRSA isolates were tested for Mupirocin resistance by Epsilometer test (E-test) using Mupirocin E-strips. A total of 186 swabs from the anterior nares were collected from HCPs, out of which 18 were positive for S. aureus. Among the 18 isolates 10 were Methicillin sensitive S. aureus (MSSA) and 8 were MRSA. The nursing staff had the highest prevalence of MRSA (50\%). None of the staff from the critical areas or treating doctors were MRSA colonisers. All the MRSA isolates were Mupirocin sensitive. The repeat swabs taken from MRSA positive group after 1 week of Mupirocin therapy were all negative for $S$. aureus. The HCPs with nasal colonisation of MRSA are potential source for health care associated infections. Mupirocin is used currently as a decolonising agent for MRSA. Therefore monitoring the emergence of Mupirocin resistance as well as ongoing training classes on infection control practices like hand hygiene and standard precautions are mandatory for prevention and control of infections.
\end{abstract}

\section{Introduction}

Staphylococcus aureus (S. aureus) and its resistant form Methicillin-resistant S.aureus (MRSA) is now one of the most prevalent and clinically significant pathogen causing health care associated infections. Nasal carriage of $S$. aureus among health care providers (HCPs) plays a key role in the epidemiology and pathogenesis of such infections (Pai et al., 2010). Colonised or infected HCPs may serve as reservoir and disseminator of MRSA not only to the hospitalised patients but also to the community at large (Thilakvathy et al., 2015). Mupirocin is a topical Glycopeptide antibiotic agent that specifically binds to bacterial isoleucyl-tRNA synthetase (IRS) and inhibits protein synthesis. The increase use of 
Mupirocin to treat Staphylococcal infections and colonisation has led to the emergence of resistant strains. Mupirocin resistance is categorised into two types: Low-level or intermediate resistance (MupL or MupI), with minimum inhibitory concentration (MICs) of 8-256 $\mu \mathrm{g} / \mathrm{ml}$, and high-level resistance (MupH), with MICs $\geq 512 \mu \mathrm{g} / \mathrm{ml}$ (Kaur and Narayan, 2014).Various studies have suggested that MupL nasal isolates can be controlled with Mupirocin therapy. Whereas, high-level resistant strains are frequently associated with failure of decolonization (Hudson, 1994; Agarwal et al., 2015).

Resistance of MRSA to Mupirocin is an emerging infection control problem which could mean potential loss of the major treatment option for controlling MRSA. Therefore this study was conducted to estimate the prevalence of nasal carriage of MRSA and its mupirocin resistance in HCPs in a rural area.

\section{Materials and Methods}

This prospective study was carried out by collecting nasal swabs from HCPs comprising of doctors, nurses, lab technologists and house-keeping staff working in Akash Hospital, Devanahalli Bangalore Rural from February to June 2017. Ethical clearance from the Institutional Ethical committee was obtained before the commencement of the study. Informed written consent was taken from all the staff who participated in the study. Health care providers who refused to give informed consent for the study and those with history of upper respiratory tract infection, recent nasal surgery, use of nasal medication, or antibiotic therapy were excluded from the study.

Sterile cotton swabs moistened with sterile normal saline were used to collect the specimen by simply rotating the swabs gently five times in both the nares. The swabs were transported immediately to the Microbiology laboratory for processing and cultured on mannitol salt agar and incubated at $37^{\circ} \mathrm{C}$ for 24 hrs. Based on the colony morphology (yellow colour mannitol fermenting colonies), Gram stain, catalase test and coagulase test, preliminary identification of $S$. aureus was made (Winn et al., 2006).Methicillin resistance was tested in Mueller Hinton agar using Cefoxitin disc (30ug), Himedia ${ }^{\circledR}$, by Kirby Bauer disc diffusion method. A zone of $\leq 21 \mathrm{~mm}$ was considered as MRSA. All the MRSA isolates were then tested for Mupirocin resistance by Epsilometer test (E-test) using Mupirocin E-strip, Himedia ${ }^{\circledR}$. Isolates with Minimum inhibitory concentration (MICs) $\geq 512 \mu \mathrm{g} / \mathrm{ml}$ were considered as high-level resistant (MupH), those with MICs 8-256 $\mu \mathrm{g} / \mathrm{ml}$ were considered as low-level resistant (MupL), and with $\leq 4 \mu \mathrm{g} / \mathrm{ml}$ were considered as Mupirocin sensitive as perClinical and Laboratory Standards Institute guidelines2016.

All the HCPs who were positive for MRSA were treated with $2 \%$ Mupirocin ointment twice daily for 5 days and were instructed to adopt strict hand hygiene and standard precautions during their routine work.

A repeat swab from the anterior nares was collected after a week to ensure decolonisation (Siegel et al., 2006). The data was analysed using SPSS version 22 and the Chi-square test was determined to check the statistical association between two categorical variables. A $\mathrm{p}$ value $<0.05$ was considered statistically significant.

\section{Results and Discussion}

A total of 186 swabs from the anterior nares were collected from the HCPs (Figure 1), out of which $18(9.68 \%)$ were positive for $S$. aureus. Among the 18 isolates 10 (5.38\%) were Methicillin sensitive S. aureus (MSSA) 
and $8(4.30 \%)$ were MRSA (Table 1$)$. The 10 MSSA were isolated from 4 nurses, 3 doctors (surgeons), 2 housekeeping staff and 1 technologist. The 8 MRSA were isolated from 4 nurses, 2 technologists and 2 housekeeping staff (Table 2). None of the MRSA isolates were from the staff in the critical areas or treating doctors. Mupirocin E-test was done on MRSA isolates (all had a MIC $\leq 4 \mu \mathrm{g} / \mathrm{ml}$ ) and all 8 were Mupirocin sensitive. The repeat swabs taken after 1 week of Mupirocin therapy were negative for $S$. aureus. There was no statistically significant association $(p>0.05)$ between nasal colonisation and gender as well as MRSA colonisation and various professional groups (Table 1 and 2).

In our study the nasal carriage of $S$. aureus was $9.68 \%$ out of which MRSA carriage was only $4.30 \%$. Both of which were similar to the study conducted by Thilakavathy et al., (2015) and Goyal et al., (2002). The highest prevalence was among the nurses from the wards $(50 \%)$ followed by housekeeping staff $(25 \%)$ and technologist $(25 \%)$ which was similar to the study conducted by Thilakavathy et al., (2015), Kaur and Narayan (2014) and Agarwal et al., (2015).

Table.1 Positive isolates and their Mupirocin susceptibility ( $\mathrm{N}=186)$

\begin{tabular}{|c|c|c|c|c|c|}
\hline Isolates & Male & Female & Total & P Value & $\begin{array}{c}\text { Mupirocin } \\
\text { susceptibility }\end{array}$ \\
\hline MSSA & $3(1.61 \%)$ & $7(3.76 \%)$ & $10(5.38 \%)$ & \multirow{3}{*}{$\mathrm{p}>0.05^{*}$} & \multirow{3}{*}{ Sensitive } \\
\hline MRSA & $2(1.07 \%)$ & $6(3.22 \%)$ & $8(4.30 \%)$ & & \\
\hline $\begin{array}{c}\text { Total no of } \\
\text { S.aureus isolated }\end{array}$ & $5(2.68 \%)$ & $13(6.98 \%)$ & $18(9.68 \%)$ & & \\
\hline
\end{tabular}

*Chi-square- Association between Gender and Colonisation

Table.2 Distribution of MRSA among HCPs and their respective departments (N=186)

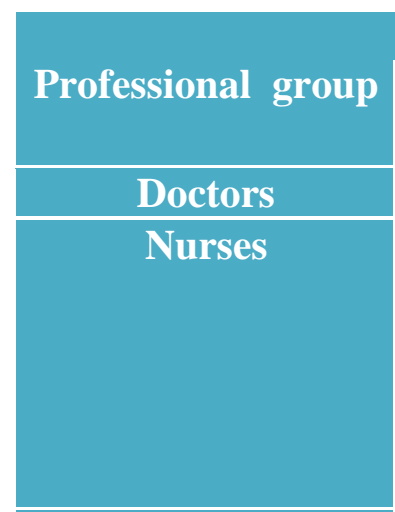

Technologists

Housekeeping staff

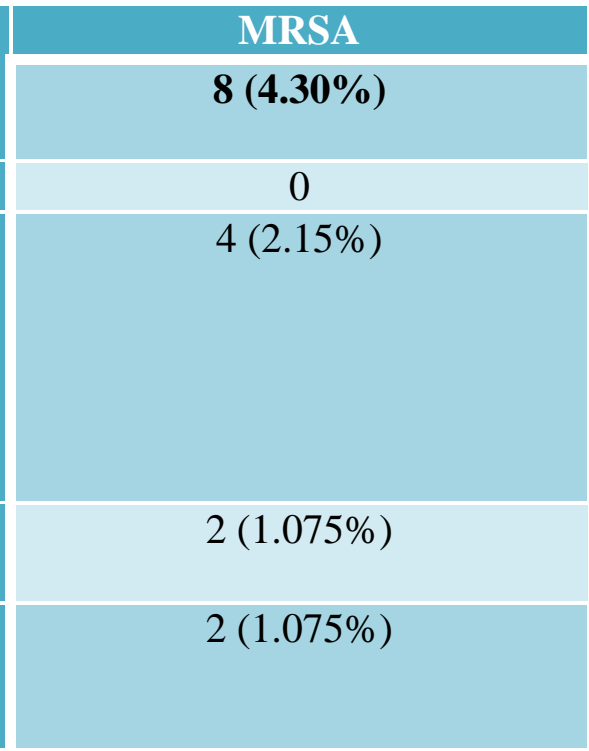

*Chi-square- Association between Profession and MRSA Colonisation

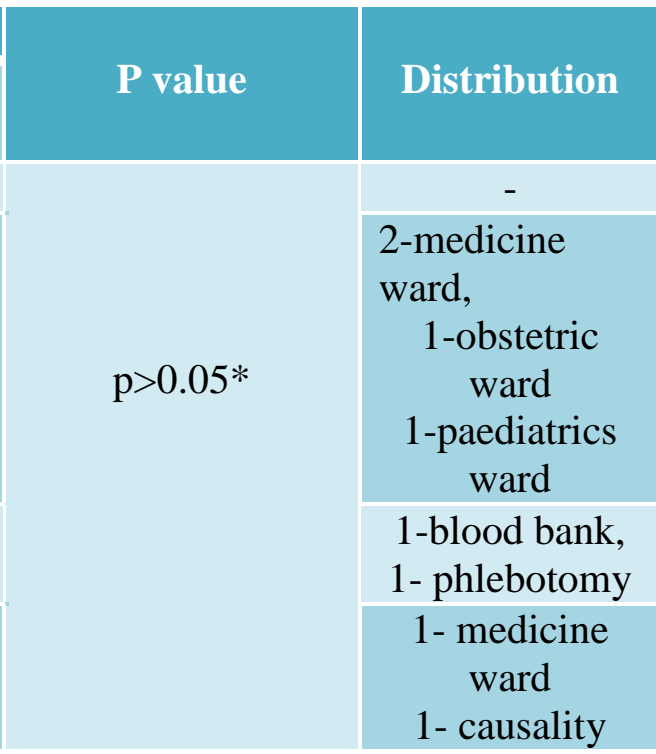

1- causality 


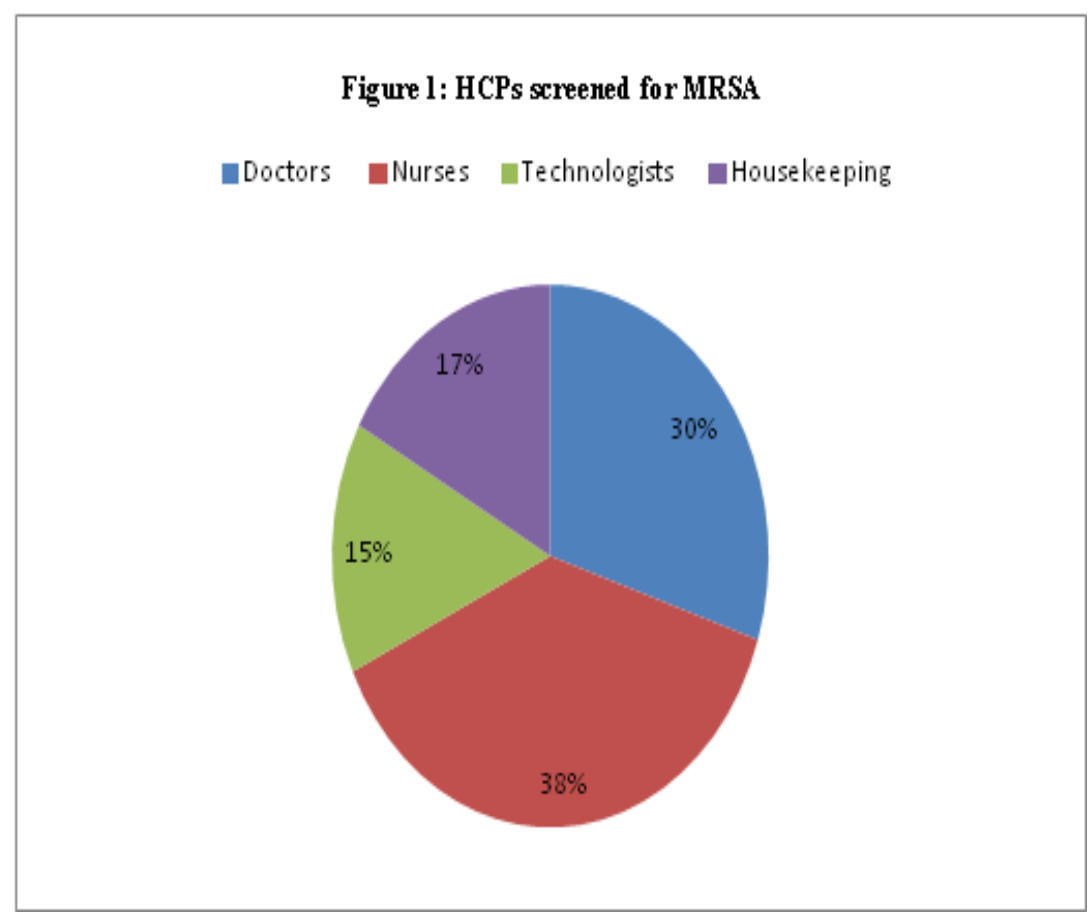

The $S$. aureus and MRSA colonisation rate is much less compared to other studies (Kaur and Narayan, 2014; Agarwal et al., 2015; Mathanraj et al., 2009) in India which could be because of the low local prevalence of MRSA in rural area, which was estimated to be only $5.03 \%$ (MRSA infection rate) in a recent study conducted by Thomas et al., (2016) in the same hospital in 2015-2016. The prevalence was probably more in nurses from the wards as they are the personnel closely associated with infected patients. Most of them that is 3 student nurses, 1 technologist and 1 housekeeping staff who were MRSA colonisers had not undergone our induction classes on infection control practices.

All the 8 MRSA were Mupirocin sensitive which was similar to study conducted by Kaur and Narayan (2014), Thilakavathy et al., (2015), and Oommen et al., (2010).

Health care providers with nasal colonisation of MRSA are potential source of health care associated infections. Our hospital being a new hospital there is regular entry of new staff, therefore routine screening and decolonisation should be made mandatory to prevent the spread of infection. Mupirocin is currently used as a decolonising agent, especially for the anterior nares. Hence the estimation of Mupirocin resistance is very important so that an alternative option like chlorhexidine can be introduced if its resistant. Induction classes before the new staff start work and thereafter ongoing training classes on infection control practices like hand hygiene and standard precautions should be conducted as a measure to curb the spread of infection.

\section{Acknowledgments}

We would like to thank Dr. Ipsita Debata and Mrs. Soumya B Shetty from the Department of Community- Medicine for their help in statistical analysis of the data

\section{References}

Agarwal L, Singh AK, Sengupta C, Agarwal A. Nasal carriage of methicillin and Mupirocin resistant S.aureus among health care workers in a tertiary care 
hospital. J Res Pharm Pract 2015; 4:182-6.

Goyal R, Das S, Mathur M. Colonisation of methicillin resistant Staphylococcus aureus among health care workers in a tertiary care hospital of Delhi. Indian J Med Sci 2002; 56:321-4.

Hudson IR. The efficacy of intranasal mupirocin in the prevention of staphylococcal infections: A review of recent experience. $J$ Hosp Infect. 1994; 27:81-98.

Kaur DC, Narayan PA. Mupirocin resistance in nasal carriage of Staphylococcus aureus among healthcare workers of a tertiary care rural hospital. Ind $J$ Crit Care Med 2014; 18: 716-21.

Mathanraj S, Sujatha S, Sivasangeetha K, Parija SC. Screening for methicillinresistant Staphylococcus aureus carriers among patients and health care workers of a tertiary care hospital in south India. Indian J Med Microbiol 2009; 27:62-4.

National Committee for Clinical Laboratory Standards. Performance standards for antimicrobial susceptibility testing; 26th informational supplement Wayne, PA: National Committee for Clinical Laboratory Standards; 2016 (M100S26).

Oommen SK, Appalaraju B, Jinsha K. Mupirocin resistance in clinical isolates of staphylococci in a tertiary care centre in South India. Indian J Med Microbiol 2010; 28:372-5.

Pai V, Rao VI, Rao SP. Prevalence and Antimicrobial Susceptibility Pattern of
Methicillin resistant Staphylococcus aureus [MRSA] Isolates at a Tertiary Care Hospital in Mangalore, South India. J Lab Physicians 2010; 2(2):8284.

Siegel JD, Rhinehart E, Jackson M, Chiarello L. The Healthcare Infection Control Practices Advisory Committee. Management of Multidrug-Resistant Organisms in Healthcare Settings; 2006. Available from: http://www.cdc.gov/ncidod/dhqp/pdf/ar/ mdro Guideline 2006.pdf.

Thilakavathy P, Vijaykumar GS, Ramesh A, Janagond $A B$, Rajendran $\mathrm{T}$, Jeremiah SS, Vithiya G. Methicillin-resistant Staphylococcus aureus Nasal Carriage Among Health-care Workers: Decolonization and Follow-up Study Conducted in a Tertiary Care Hospital. J Hum Health 2015; 1:16-9

Thomas R, Shilpa K, Ramyashree R. Prevalence and Recent Trend in the Antibiogram of Staphylococcus Species Isolated from Clinical Specimens in a Rural Tertiary Care Hospital in South India Int.J.Curr.Microbiol.App.Sci 2016 5(12): 644-649.

Winn WC, Allen SD, Janda WM, Koneman EW, Procop GW, Schreckenberger PC, Woods G. Chapter 12 Gram Positive Cocci. Part I: Staphylococci and Related Gram Positive Cocci. 6th. Philadelphia: Lippincott Williams and Wilkins; 2006. Color atlas and textbook of diagnostic microbiology. Pp. 623-671.

\section{How to cite this article:}

Sangeetha, K.T., S. Sreeja and Ruby Thomas. 2018. A Prospective Study Estimating the Mupirocin Resistance in Health Care Providers with MRSA Nasal Carriage in a Tertiary Care Hospital in Rural South India. Int.J.Curr.Microbiol.App.Sci. 7(01): 659-663. doi: https://doi.org/10.20546/ijcmas.2018.701.080 